\title{
Management of the Contralateral Neck in Oral Squamous Cell Carcinoma in the UK and a Review of Literature
}

\section{Garg $M^{1 *}$, Alibhai $\mathbf{M}^{2}$ and Bisase $B^{1}$}

${ }^{1}$ Department of Oral and Maxillofacial Surgery, Queen

Victoria Hospital NHS Foundation Trust, UK

${ }^{2}$ Department of Oral and Maxillofacial Surgery, University College Hospital, UK

*Corresponding author: Montey Garg, Department of Oral and Maxillofacial Surgery, Queen Victoria Hospital NHS Foundation Trust, UK

Received: February 04, 2019; Accepted: February 27, 2019; Published: March 06, 2019

\begin{abstract}
Oral squamous cell carcinoma (OSCC) has a variable incidence of contralateral neck (CLN) metastases which affects long-term survival and prognosis. Therefore, due consideration should be given to the management of the CLN in OSCC for certain cases. Neck dissection is often indicated in the NO cases where the primary OSCC obviously crosses the midline. However, there is much variability in the management of the CLN when considering two other clinical events encountered in multidisciplinary meetings.
\end{abstract}

These were interrogated by conducting an online survey in the UK. The first scenario included a tumour close to but not crossing the midline and the second included an ipsilateral metachronous or recurrent tumour when the ipsilateral neck (ILN) had been previously treated. The respondents included head and neck $(\mathrm{H} \& N)$ consultant clinicians in the UK.

Our findings suggest wide inconsistencies in the management of the CLN in these particular situations. The variability in practice is also reflected in the literature review. There is a need to address the CLN. There may be a role for sentinel lymph node biopsy (SLNB) in staging the neck for tumours close to the midline or in ipsilateral metachronous tumour or recurrence in a patient with a previously treated ILN.

Keywords: CLN; OSCC; H\&N

\section{Introduction}

OSCC is the most frequent of H\&N malignancies [1]. The most significant prognostic and survival factor in patients with OSCC is neck lymph node metastasis [2].

OSCC has a high incidence of cervical micro metastases and lateralised tumours can sometimes metastasise contralaterally because of the rich lymphatic intercommunications relative to submucosal plexus of oral cavity that freely communicates across the midline. Consequently, the neoplastic cells can spread to any area of the neck [1].

Despite several retrospective studies and review papers in the literature about the risk factors predicting CLN metastasis, the management of the CLN in OSCC remains controversial in certain scenarios. Two such situations arise when firstly, a tumour is close to but does not cross the midline, and secondly in ipsilateral metachronous or recurrent tumours when the ILN has previously been treated.

We conducted a survey to explore the current management of the CLN in OSCC in the UK based on these scenarios.

\section{Methods}

We conducted an online survey regarding the management of the CLN and distributed the survey via email to over $150 \mathrm{H} \& \mathrm{~N}$ consultant clinicians (Surgeons and oncologists) around the UK targeting as many multidisciplinary teams as possible.

The survey had five questions.

\section{Results}

The results are presented with the question above and the answer received (Figures 1-5).

53 responses were received from Consultants in OMFS, ENT, Plastics and Oncology. Figure 1 shows the percentage response from Consultants in different specialties.

Figure 2 shows that all respondents were of consultant grade.

The third question (Figure 3 ) suggests a resection past the midline

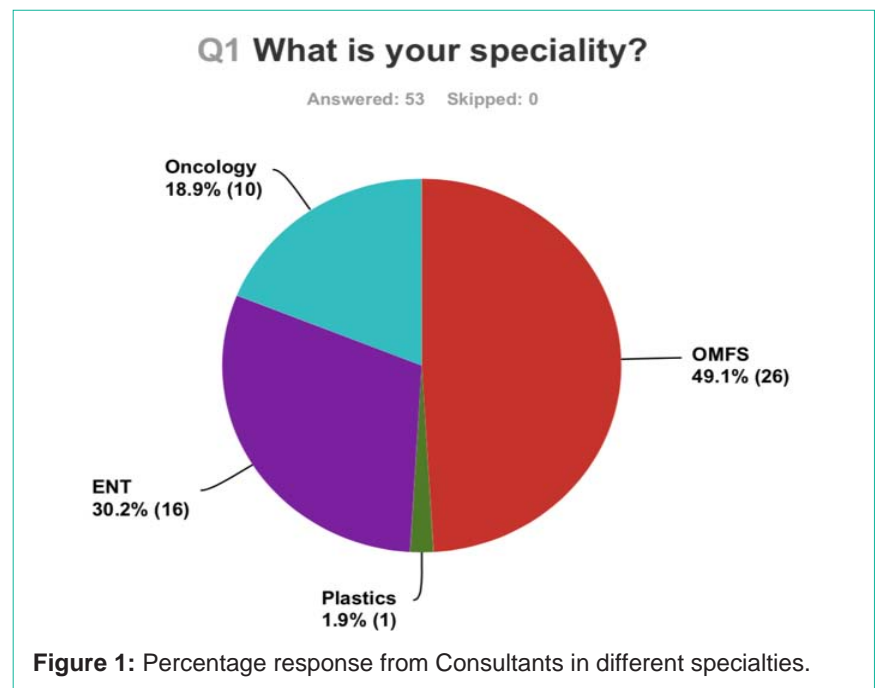

Austin J Surg - Volume 6 Issue 5 - 2019

ISSN : 2381-9030 | www.austinpublishinggroup.com

Garg et al. () All rights are reserved
Citation: Garg M, Alibhai M and Bisase B. Management of the Contralateral Neck in Oral Squamous Cell Carcinoma in the UK and a Review of Literature. Austin J Surg. 2019; 6(5): 1170. 


\section{Q2 What is your current grade?}

Answered: 53 Skipped: 0

\begin{tabular}{|c|c|c|}
\hline Answer Choices & Response & \\
\hline Consultant & $100.0 \%$ & 53 \\
\hline Post CCT Fellow & $0.0 \%$ & 0 \\
\hline Pre CCT Fellow & $0.0 \%$ & 0 \\
\hline Total & & 53 \\
\hline
\end{tabular}

Figure 2: What is your current grade?

\section{Q3 A 40 year old male (PS 0) presents with a \\ T3NOMO moderately differentiated SCC of \\ the right lateral tongue after complete \\ staging. The tumour is close to but does \\ not reach or cross the midline on MR \\ imaging, however an adequate $1 \mathrm{~cm}$ \\ resection margin will cross the midline. \\ What would be your preferred \\ management?}

Answered: 53 Skipped:0

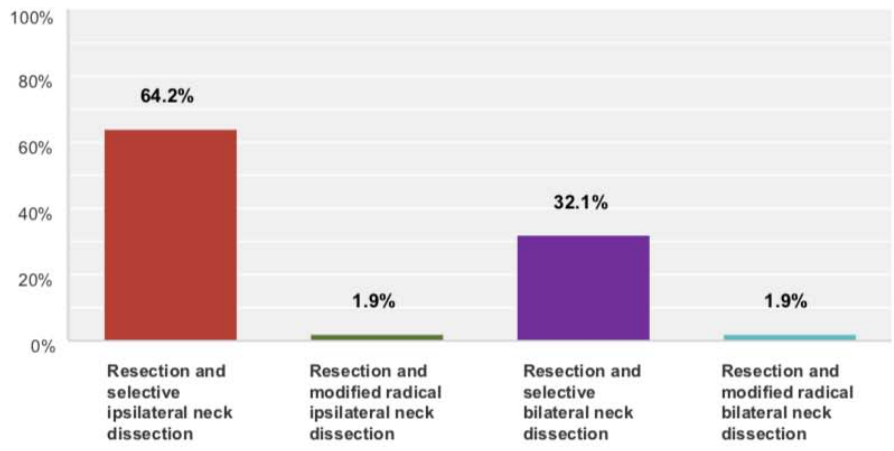

\begin{tabular}{|c|c|c|}
\hline Answer Choices & Respo & \\
\hline Resection and selective ipsilateral neck dissection & $64.2 \%$ & 34 \\
\hline Resection and modified radical ipsilateral neck dissection & $1.9 \%$ & 1 \\
\hline Resection and selective bilateral neck dissection & $32.1 \%$ & 17 \\
\hline Resection and modified radical bilateral neck dissection & $1.9 \%$ & 1 \\
\hline Total & & 53 \\
\hline
\end{tabular}

Figure 3: A 40 year old male (performance status 0) presents with a $\mathrm{T}_{3} \mathrm{~N}_{0} \mathrm{M}_{0}$ moderately differentiated SCC of the right lateral tongue after completing staging. The tumour is close to but does not cross the midline on Magnetic resonance imaging (MRI), however an adequate $1 \mathrm{~cm}$ resection margin will cross the midline. What would be your preferred management?

with $64 \%$ of clinicians offering an ipsilateral neck dissection and $4 \%$ offering modified radical neck dissection either ipsilateral or bilateral rather than selective neck dissection in the elective setting.

Figure 4 shows that nearly one-third of the clinicians reported that they would not recommend CRT (Chemoradiotherapy). Of these, only one would not offer any adjuvant treatment at all.

Responses to the last question (Figure 5) were varied with the majority favouring resection and close follow up. Nearly $20 \%$ would recommend resection and SLNB.

\section{Discussion}

Nodal metastasis is the most influential factor in the prognosis of OSCC [3]. Since work by Criles (1906), Martin (1951) and Suarez (1963) delineating the value of various neck dissections ranging from radical to modified or "functional" neck dissection, the late twentieth century saw the concept of selective neck dissection introduced where only the nodal groups at greatest risk of metastasis from a 


\section{Q4 If the contralateral (left) neck was not surgically treated (resection and ipsilateral neck dissection only) and histology shows: right tongue pT3 resection with a closest margin of $5.5 \mathrm{~mm}$, right neck $\mathrm{N} 1$ with ECS. Regarding the neck, what would be your next recommendation?}

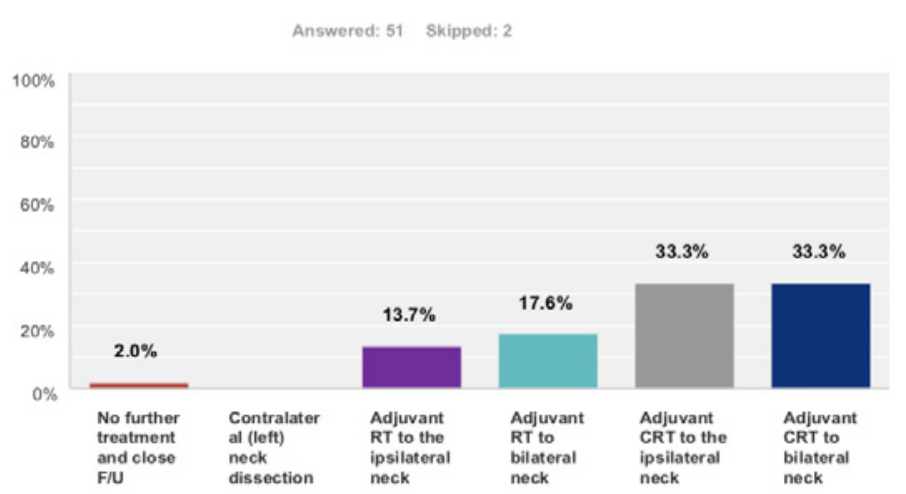

\begin{tabular}{|l|l|}
\hline Answer Choices & Responses \\
\hline No further treatment and close F/U & $2.0 \%$ \\
\hline Contralateral (left) neck dissection & $0.0 \%$ \\
\hline Adjuvant RT to the ipsilateral neck & $13.7 \%$ \\
\hline Adjuvant RT to bilateral neck & $17.6 \%$ \\
\hline Adjuvant CRT to the ipsilateral neck & $33.3 \%$ \\
\hline Adjuvant CRT to bilateral neck & $33.3 \%$ \\
\hline Total & \\
\hline
\end{tabular}

Figure 4: If the contralateral (left) neck was not surgically treated (resection and ipsilateral neck dissection only) and the histology shows: right tongue ${ }_{\mathrm{p}}{ }_{3}$ resection with a closest margin of $5.5 \mathrm{~mm}$, right neck $\mathrm{N}_{1}$ with extracapsular spread (ECS). Regarding the neck, what would be your next recommendation?

given primary site were removed [4,5]. Selective neck dissections are now widely employed for staging and in some properly selected cases, therapeutic treatment of the neck [5].

For unilateral OSCC, conventional elective or therapeutic ILN dissection is performed. It is also well known that a higher frequency of bilateral lymph node metastases is observed in patients in whom OSCC lies across the midline [6]. How does primary unilateral OSCC spread to the CLN?

Feind et al. [7] proposed three ways of contralateral spread of metastases in the H\&N. Firstly through crossing lymphatic afferent vessels, secondly by actual spread over the midline via efferent lymph vessels after regional nodes become extensively involved and collateral lymphatic flow takes place and finally in certain anatomic areas in the $\mathrm{H} \& \mathrm{~N}$ where there is no definite midline. They demonstrated that lesions from the lip, middle of the floor of mouth and base of tongue had frequent contralateral metastasis [7].

Elective CLN treatment is recommended for OSCC crossing the midline, but is not routinely performed in lateralised cases and is still a matter of debate [8].

Our scenario based survey explores if there is any consistency in managing the CLN by Consultants in the UK.

\section{First Scenario Based Question}

The first question (Figure 3) provides a few predictive factors in relation to CLN metastasis and includes TNM stage (Clinical Stage), tumour size, nodal status, histological differentiation, tumour site and proximity of the tumour to midline.

\section{Clinical Stage}

Kowalski et al. [9] found that the clinical stage (CS) was one of the most important predictors of CLN metastases in OSCC. They found that the groups of clinical stage (CS) II, III and IV had risks from 1.8 to 9.6 times higher than cases of CS I [9]. Frequency of such metastases was $33 \%$ for $\mathrm{T}_{4}, 15 \%$ for CS III, and $32 \%$ for CS IV. Risk of contralateral metastases was over $20 \%$ in stage $\mathrm{T}_{1-3} \mathrm{~N}_{2 \mathrm{a}-3}$ and $\mathrm{T}_{4} \mathrm{~N}_{0-3} \mathrm{M}_{0}$ tumours $[9,10]$. Gonzalez-Garcia et al. [11] found that $6.7 \%$ patients with staging IV of TNM classification developed CLN metastasis compared to only $2.6 \%$ of patients with TNM stage I [10,11].

\section{Tumour Size}

Kurita et al. [6] found that CLN metastases in patients with the $\mathrm{T}_{2}$ and $\mathrm{T}_{3}$ tumour occurred only in cases of mobile tongue, but not in other sites. Excluding cases of tongue SCC, CLN metastasis was unlikely in patients with $\mathrm{T}_{1}$ to $\mathrm{T}_{3}$ OSCC that occurred in the unilateral side $[6,10]$. Koo et al. [3] showed that the rate of contralateral occult 
metastasis was $8 \%$ for $\mathrm{T}_{2}, 25 \%$ for $\mathrm{T}_{3}$ and $18 \%$ for $\mathrm{T}_{4}$ and no metastasis was observed in the $\mathrm{T}_{1}$ cases $[3,10]$.

\section{Nodal Status}

Capote-Moreno et al. [12] found that $21.6 \%$ of the cases with positive homolateral nodes showed positive contralateral metastases whereas contralateral disease developed in only $6.4 \%$ cases with negative homolateral nodes. Kurita et al [6] found that the incidence of CLN metastasis was higher in patients with multinode involvement (50\%) than with those with single node involvement $(26.1 \%)[6,10]$. They reported that CLN node metastases never occurred without ILN node metastasis $[1,6,10]$. So CLN node metastasis is unlikely if ipsilateral node metastasis has not occurred. This may be possibly due to the aberrant migration of in-transit carcinomatous cells to the opposite side of the neck due to elective ILN dissection and tumour resection [1]. Olzowy et al. [13] also found that patients with two or more ILN node metastases showed significantly more bilateral metastases compared with patients with fewer than two positive ipsilateral nodes [1,13]. However, Gonzalez-Garcia et al. [14] did not find any association between the nodal status of the ILN and higher rate of CLN metastases $[10,14]$.

\section{Grade of Histological Differentiation}

Gonzalez-Garcia et al found that $13.5 \%$ of the patients with poorly differentiated SCC developed CLN metastasis compared to $5.2 \%$ with well-differentiated tumours [1,11]. Kurita et al. [6] also found that the risk for CLN metastasis increased with advanced histopathological grading $[6,10]$.

\section{Tumour Site and Proximity to the Midline}

There is no clear consensus about which location is of higher risk for CLN metastasis [10]. Martin et al. [15] reported that primary tumour invasion crossing the midline of oral cavity was associated with a higher risk of CLN metastasis $[1,10,15]$. Koo et al. [3] also found increased risk of contralateral metastasis for tumours crossing the midline [3,10]. Kowalski et al. [9] found that lesions over $1 \mathrm{~cm}$ away from the midline had a very low risk of contralateral metastases (7\%) and risk increased to $16 \%$ in cases with tumours crossing the midline by less than $1 \mathrm{~cm}$ and reached $46 \%$ in those where the crossing was more than $1 \mathrm{~cm}$ [9].

Capote-Moreno et al. [12] reported a higher tendency for contralateral metastases in tumours located in the tongue base (31.4\%) and the floor of the mouth (11\%), with a lower frequency in the mobile tongue $(7.2 \%)$ and the or opharynx $(6.3 \%)[1,12]$. Kowalski et al. [9] suggested that tumours invading the tongue and the floor of the mouth have a higher risk of contralateral metastases than invading the retromolar trigone [9]. Kurita et al. [6] interestingly reported that the incidence of CLN metastases was higher in cases of lower gum carcinoma (25\%) than those with mobile tongue carcinoma (15.4\%) [6].

\section{Management of the First Scenario Based Question}

In our scenario, the tumour being a Stage III $\mathrm{T}_{3}$ moderately differentiated lateral tongue tumour and close to the midline has a risk for CLN metastasis. $\mathrm{N}_{0}$ status of the neck does somewhat reduce the risk for contralateral metastasis.

$64.2 \%$ of the consultants offered resection and selective ILN dissection whereas $32.1 \%$ offered resection and selective bilateral neck dissection in the scenario. Perhaps, consultants offering bilateral neck dissection perceived the increased risk of occult CLN metastasis.

Fan et al. [1] summarised all indications for elective CLN dissection in oropharyngeal SCC which included- tumours crossing the midline; advanced staging $\left(\mathrm{cT}_{34}\right)$; primary tumour more than $3.75 \mathrm{~mm}$ thick; multiple ipsilateral node involvement; and tumours arising in the base of the tongue and floor of the mouth [1]. Based on these indications, $\mathrm{CT}_{3}$ meets the criteria for elective CLN dissection in our scenario. Elective CLN treatment is also acceptable for oral cancer approaching or crossing the midline [10].

On the contrary, Lanzer et al. [16] concluded that locoregional lymph node recurrence, disease free survival and overall survival rates did not show a statistically significant difference between their group $(\mathrm{n}=24)$ which underwent CLN dissection for $\mathrm{N}_{0}$ neck and the other group $(n=128)$ where CLN was observed in lateralised oral and oropharyngeal SCC [16]. Habib et al. [17] conducted another retrospective study and observed cases of $\mathrm{cN}_{0}$ CLN in lateralised OSCC. They only found a $2.9 \%$ rate of isolated CLN failure. González-García et al. [11] in their study found that only 2 out of 64 patients undergoing bilateral neck dissection as definitive treatment developed CLN metastases whereas 14 of the 149 patients undergoing ILN dissection developed CLN metastases. Despite this, they recommended bilateral neck dissection in selected patients (due to the added morbidity) with tumours arising in the midline [11]. Feng et al. [18] concluded that for patients with low and moderate risk of contralateral metastasis, observation should be sufficient if strict compliance with a cancer surveillance protocol is followed [18]. The variability in management of the CLN as reflected in our survey is echoed in the literature review.

\section{Second Scenario Based Question}

The second scenario based question (Figure 4) follows on from the first scenario. The CLN is not treated in the first scenario and the tumour is resected with ILN dissection. The histology confirms a $\mathrm{pT}_{3} \mathrm{~N}_{1}$ tumour with a closest margin of $5.5 \mathrm{~mm}$. There is Extra Capsular Spread (ECS) noted in the ILN. What will be the further management?

Feng et al. [10,18] and Liao et al. [8,10] have concluded that ECS is co-related with CLN metastasis. Furthermore, less than $1 \mathrm{~cm}$ of non-affected tissue around the tumour increases the risk of CLN metastasis [10]. This scenario explores whether UK clinicians will offer adjuvant radiotherapy or adjuvant CRT to ipsilateral or bilateral neck. A third will offer adjuvant CRT to bilateral neck, a third will offer CRT to ILN only and nearly a third will not offer CRT of which one would not offer any adjuvant treatment at all. Bernier et al. [19] have shown that in locally advanced $\mathrm{H} \& \mathrm{~N}$ cancer, microscopically involved resection margins and ECS of tumour from neck nodes are the most significant prognostic factors for poor outcome. The addition of concomitant cisplatin to postoperative radiotherapy improves outcome in patients with one or both of these risk factors [19]. The EORTC (European Organisation Research and Treatment of Cancer) and RTOG (Radiation Therapy Oncology Group) trials 


\section{Q5 The contralateral (left) neck was not treated and only the ipsilateral (right) neck was treated with selective neck dissection and adjuvant therapy. 18 months later the patient is staged with a T3NOMO ipsilateral (right) metachronous buccal mucosa SCC. What would be your preferred management?}

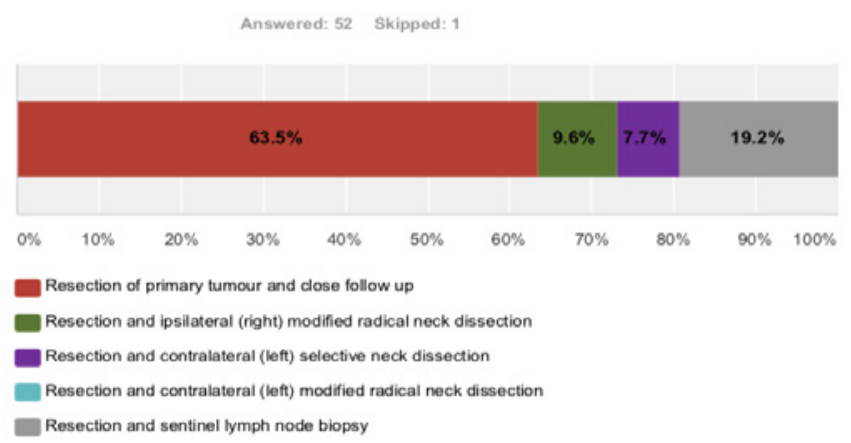

\begin{tabular}{|l|l|}
\hline Answer Choices & \multicolumn{1}{|c|}{ Responses } \\
\hline Resection of primary tumour and dose follow up & $\mathbf{6 3 . 5 \%}$ \\
\hline Resection and ipsilateral (right) modified radical neck dissection & $\mathbf{9 . 6 \%}$ \\
\hline Resection and contralateral (left) selective neck dissection & $\mathbf{7 . 7 \%}$ \\
\hline$\quad$ Resection and contralateral (left) modified radical neck dissection & $\mathbf{0 . 0 \%}$ \\
\hline$\quad$ Resection and sentinel lymph node biopsy & $\mathbf{1 9 . 2 \%}$ \\
\hline Total & \multicolumn{1}{|c|}{0} \\
\hline
\end{tabular}

Figure 5: The contralateral (left) neck was not treated and only the ipsilateral (right) neck was treated with selective neck dissection and adjuvant therapy. 18 months later, the patient is staged with a $\mathrm{T}_{3} \mathrm{~N}_{0} \mathrm{M}_{0}$ ipsilateral (right) metachronous buccal mucosa SCC. What would be your preferred management?

have also provided evidence of improved clinical outcomes with CCRT in patients with positive margins and ECS of tumour from neck nodes $[20,21]$. Thus, the responses of two-third of the clinicians in our survey are in keeping with the above research findings and a third of these clinicians would also treat the CLN possibly due to ECS and close margins.

\section{Third Scenario Based Question}

This question partly explores the risk of CLN metastases in OSCC with reported rates between 0.9-36\% [10] but mainly challenges awareness of altered patterns of drainage of the neck in a previously treated patient. The possibility of a higher risk of CLN metastases despite a well lateralised recurrent ipsilateral tumour is raised. There is a paucity in the literature about the drainage pattern in the neck after ipsilateral surgery, surgery and radiotherapy/CRT or radiotherapy/ CRT alone. Nodal basins in the event of an ipsilateral metachronous or recurrent tumour could include the CLN, retropharyngeal nodes, central non-irradiated laryngeal strip or undissected ipsilateral basins such as Robbins level IIB, IV or V. Responses were varied with the majority favouring resection and close follow up. Interestingly, 20\% would recommend resection and SLNB.

The literature is also devoid of studies that discuss where neck failures occurred in metachronous or recurrent ipsilateral disease. Albeit an infrequent event, a sequel to this paper will be looking at our cohort of patients in whom the ipsilateral recurrent or metachronous disease has occurred and have either failed in the neck at various sites or had neck surgery with relevant findings pertinent to this paper.

Schilling et al. [22] in the Sentinel European Node Trial (SENT) found that lateral tumours in oral cavity SCC drained ipsilaterally in $87 \%$ of the cases ( 320 in 369 ) but in $10 \%$ (40 cases) they drained bilaterally and in $2.4 \%$ (9 cases) exclusively to the CLN [22]. The role of SLNB in the third scenario will be useful in identifying nodal disease in case of metachronous tumour with previously treated ILN.

Some may question the value of determining a retropharyngeal echelon sentinel node as traditionally it is not routine to dissect this area. We would argue that it may improve local control and possible long term outcome if this node was removed for staging or therapeutic value if it was the only positive nodal basin. We suggest this as with the advent of robotic surgery in the oropharynx, this previously avoided area is now more accessible using such innovative techniques today.

\section{Conclusion}

The surgical management of the CLN in OSCC approaching the midline and that needing adjuvant treatment is not uniform in this cohort of clinicians. This is reflected in our literature review too. The management of the CLN in the event of an ipsilateral metachronous or recurrent tumour is also heterogenous in this group. The merits of SLNB in staging the neck have been shown in early OSCC. We believe there may be a role for SLNB in staging the neck in these scenarios. 


\section{References}

1. Fan S, Tang Q, Lin Y, Chen W, Li J, Huang Z, et al. A review of clinical and histological parameters associated with contralateral neck metastases in oral squamous cell carcinoma. Int J Oral Sci. 2011; 3: 180-191.

2. Donaduzzi LC, De-Conto F, Kuze LS, Rovani G, Flores ME, Pasqualotti A Occurrence of contralateral lymph neck node metastasis in patients with squamous cell carcinoma of the oral cavity. J Clin Exp Dent. 2014; 6: e20913.

3. Koo BS, Lim YC, Lee JS, Choi EC. Management of contralateral NO neck in oral cavity squamous cell carcinoma. Head Neck. 2006; 28: 896-901.

4. Crile GW. Excision of cancer of the head and neck. JAMA. 1906; 47: 1780 1786.

5. Ferlito A, Rinaldo A, Silver CE, Shah JP, Suárez C, Medina JE, et al. Neck dissection: then and now. Auris Nasus Larynx. 2006; 33: 365-374.

6. Kurita H, Koike T, Narikawa J, Sakai H, Nakatsuka A, Uehara S, et al. Clinica predictors for contralateral neck lymph node metastasis from unilateral squamous cell carcinoma in the oral cavity. Oral Oncol. 2004; 40: 898-903.

7. Feind $\mathrm{CR}$, Cole RM. Contralateral spread of head and neck cancer. Am J Surg. 1969; 118: 660-665.

8. Liao CT, Huang SF, Chen IH, Chang JT, Wang HM, Ng SH, et al. Risk stratification of patients with oral cavity squamous cell carcinoma and contralateral neck recurrence following radical surgery. Ann Surg Oncol. 2009; 16: 159-170.

9. Kowalski LP, Bagietto R, Lara JR, Santos RL, Tagawa EK, Santos IR. Factors influencing contralateral lymph node metastasis from oral carcinoma. Head Neck. 1999; 21: 104-110.

10. Villanueva-Alcojol L. Contralateral neck dissection in oral squamous cell carcinoma: when it should be done? Plast Aesthet Res. 2016; 3: 181-188.

11. González-García R, Naval-Gías L, Rodríguez-Campo FJ, Sastre-Pérez J, Muñoz-Guerra MF, Gil-DíezUsandizaga JL. Contralateral lymph neck node metastasis of squamous cell carcinoma of the oral cavity: a retrospective analytic study in 315 patients. J Oral Maxillofacial Surg. 2008; 66: 1390-1398.

12. Capote-Moreno A, Naval L, Muñoz-Guerra MF, Sastre J, Rodríguez- Campo FJ. Prognostic factors influencing contralateral neck lymph node metastases in oral and oropharyngeal carcinoma. J Oral Maxillofac Surg. 2010; 68: 268 275
13. Olzowy B, Tsalemchuk Y, Schotten KJ, Reichel O, Harréus U. Frequency of bilateral cervical metastases in oropharyngeal squamous cell carcinoma: a retrospective analysis of 352 cases after bilateral neck dissection. Head Neck. 2011; 33: 239-243

14. González-García R, Naval-Gías L, Sastre-Pérez J, Rodriguez-Campo FJ Muñoz-Guerra MF, Gil-Diez Usandizaga JL, Diaz-Gonzalez FJ. Contralatera lymph neck node metastasis of primary squamous cell carcinoma of the tongue: a retrospective analytic study of 203 patients. Int J Oral MaxillofacSurg. 2007; 36: 507-513.

15. Martin H, Del Valle B, Ehrlich H, Cahan WG. Neck dissection. Cancer. 1951; 4: $441-499$

16. Lanzer M, Zemann W, Lubbers TH, Kruse A, Reinisch S. Do patients with oral and oropharyngeal squamous cell carcinoma benefit from elective contralateral neck dissection? A long-term analysis. Head Neck Oncol. 2012; 4: $70-75$.

17. Habib M, Murgasen J, Gao K, Ashford B, Shannon K, Ebrahimi A. Contralateral neck failure in lateralized oral squamous cell carcinoma. ANZ J Surg. 2016; 86: 188-192.

18. Feng Z, Niu LX, Yuan Y, Peng X, Guo CB. Risk factors and treatment of contralateral neck recurrence for unilateral oral squamous cell carcinoma: a retrospective study of 1482 cases. Oral Oncology. 2014; 50: 1081-1088.

19. Bernier J, Cooper JS, Pajak TF, van Glabbeke M, Bourhis J, Forastiere A Defining risk levels in locally advanced head and neck cancers: a comparative analysis of concurrent postoperative radiation plus chemotherapy trials of the EORTC and RTOG. Head Neck. 2005; 27: 843-850.

20. Cooper JS, Pajak TF, Forastiere AA, Jacobs J, Campbell BH, Saxman SB. Postoperative concurrent radiotherapy and chemotherapy for high-risk squamous-cell carcinoma of the head and neck. Radiation Therapy Oncology Group 9501/Intergroup. N Engl J Med. 2004; 350: 1937-4194.

21. Bernier J, Domenge C, Ozsahin M, Matuszewska K, Lefèbvre JL, Greiner $\mathrm{RH}$, et al. European Organization for Research and Treatment of Cancer Trial 22931. Postoperative irradiation with or without concomitant chemotherapy for locally advanced head and neck cancer. N Engl J Med. 2004; 350: 19451952.

22. Schilling C. Sentinel European Node Trial (SENT): 3-year results of sentine node biopsy in oral cancer. Eur J Cancer. 2015; 51: 2777-2784.
Austin J Surg - Volume 6 Issue 5 - 2019

ISSN : 2381-9030 | www.austinpublishinggroup.com

Garg et al. () All rights are reserved
Citation: Garg M, Alibhai M and Bisase B. Management of the Contralateral Neck in Oral Squamous Cell Carcinoma in the UK and a Review of Literature. Austin J Surg. 2019; 6(5): 1170 